\title{
In the Guidelines for Physicians and Urologists "Watchful Waiting" for Benign Prostatic Hyperplasia (BPH) should be Replaced with Thermobalancing Therapy that Treats Prostate Effectively, Cost-Effectively and Safely
}

\author{
Simon Allen* \\ Allen S, Director, Fine Treatment, Oxford, United Kingdom
}

Received: 濫: October 20, 2018; Published: 監: October 29, 2018

*Corresponding author: Allen Simon, Director, Fine Treatment, 29 Rewley Road, Oxford, OX1 2RA, United Kingdom

\begin{abstract}
Primary care physician is the first contact for screening for benign prostatic hyperplasia (BPH), conducting timely diagnostic work, and initiating relevant therapy. To protect men with lower urinary tract symptoms (LUTS) due to BPH from severe side effects and complications after standard BPH drugs and surgeries "watchful waiting" was introduced. Innovative Thermobalancing therapy with Dr Allen's therapeutic device, patented in the USA, has demonstrated the effectiveness of treatment of LUTS due to BPH by reducing the size of the enlarged prostate, urination symptoms and improving the quality of life. The results of 10-year follow-up and clinical trials confirm that there are no side effects after using Dr. Allen's device. Therefore, the guidelines for BPH treatment and management should be amended, and "watchful waiting" must be changed to Thermobalancing therapy.
\end{abstract}

Keywords: Aging Men; Watchful Waiting; BPH Treatment; Lower Urinary Tract Symptoms; Thermobalancing Therapy; Enlarged Prostate

Abbreviations: BPH: Benign Prostatic Hyperplasia; LUTS: Lower Urinary Tract Symptoms; TT: Thermobalancing Therapy; DATD: Dr Allen's Therapeutic Device; AUA: American Urological Association; QoA: Quality of Life; CP: Chronic Prostatitis; CPPS: Chronic Pelvic Pain Syndrome; EAU: European Association of Urology;

\section{Introduction}

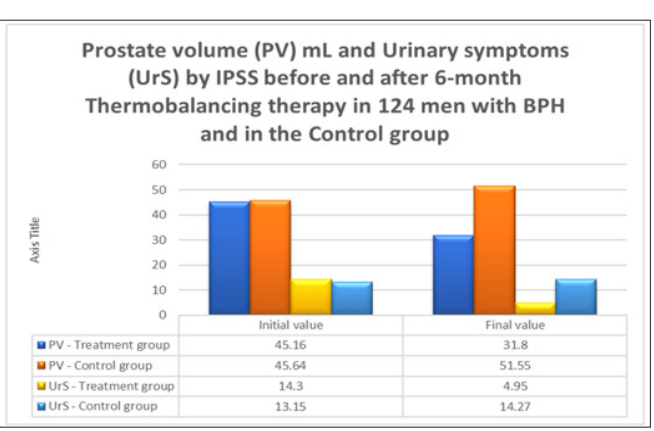

Figure 1: Prostate volume measured by ultrasound decreased from $45.1 \mathrm{~mL}$ to $31.8 \mathrm{~mL}$ and the level of urinary symptoms by IPSS decreased from 14.3 to 4.95 in 124 men with BPH after Thermobalancing therapy. In the control group prostate volume increased and urinary symptoms scores worsened. Hence DATD reduces PV and decreases urinary symptoms significantly.
Benign prostatic hyperplasia (BPH) is a common cause of lower urinary tract symptoms (LUTS) in aging men, worsening their quality of life, and physicians are positioned well to screen for BPH and to manage it [1]. Thus, primary care physician has an important role with the identification and early treatment of bothersome urinary symptoms caused by BPH [2]. It is important as the prevalence of LUTS/BPH increases with age, and the burden on the healthcare system and society may increase due to the ageing population [3]. BPH is a nonmalignant enlargement of the prostate, therefore it requires safe treatment to alleviate symptoms, delay disease progression, and lessen the chance of needing surgery for BPH. The results of 10-year follow-up and a clinical trial on Thermobalancing therapy (TT) with Dr Allen's therapeutic device (DATD) in 124 men with BPH have shown its effectiveness to treat urinary symptoms due to prostate enlargement. According to this clinical study, after use of DATD as mono-therapy the decrease in urine symptoms, measured by International Prostate Symptom 
Score, was accompanied by a significant decrease in the volume of the prostate gland, see Figure 1 [4]. It should be noted that the use of DATD improves of the quality of life (QoL) in observed men. In addition, DATD provides a new safe natural treatment method, patented is the USA [5].

\section{Watchful Waiting}

Guidelines for the management of benign prostatic hyperplasia (BPH), according to the European Association of Urology (EAU), American Urological Association (AUA) and urological associations in other countries, includes pharmacotherapy, "watchful waiting", surgical options and minimally invasive procedures [6]. The main reason for having "watchful waiting" for BPH is to protect men from severe side effects and complications after standard BPH drugs: alpha-blockers and inhibitors of the enzyme 5 alpha-reductase [7]; and surgeries [8]. For many years in "watchful waiting" are commonly used herbal medicines, such as Serenoa repens, Pygeum africanum, Urtica dioica, and dietary supplements and nutraceuticals, such as vitamin D, antioxidants, etc.

Despite widespread consumption, there is limited evidence of health benefits related to nutraceutical or supplement use and some of these products have the potential to produce significant toxicity [9]. Diet and physical activity are now considered important factors affecting prostate health in the aging male, However, whether physical exercise and modifications of dietary habit can really alter the natural history of BPH/LUTS remains to be determined [10]. The aim of this review is to summarize the management of BPH and is designed to aid the generalist with the pertinent information needed to provide excellent care for LUTS due BPH - the most common disorder for aging men.

\section{New Understanding of Etiology and Pathophysiology of BPH}

In the past decade, significant progress has been made in understanding the etiology and pathophysiology of BPH. It is becoming increasingly clear that treating the cause of BPH will lead to targeted therapy and will be useful for men who want to take a prophylactic approach. Thermobalancing therapy is based on a new understanding of the Origin of Diseases, which states: all chronic internal diseases have the same root, namely, a pathological activity of capillaries [11]. The development of prostate non-malignant chronic conditions, such as chronic prostatitis/chronic pelvic pain syndrome (CP/CPPS) and prostate enlargement or BPH, are also linked to the changes at the micro-vascular level [12]. The role of vascular dysfunction as possible mechanism of development of BPH leading to prostatic hypoxia, pelvic ischemia and increased pressure in the prostate gland were discussed by different researches [13-15].

According to the new understanding of the cause of BPH, the pathological activity of capillaries is in the core of the development of prostate enlargement, which is based on two functions of small blood vessels, namely constriction and spontaneous expansion of capillaries. Constriction of capillaries in response to different triggers, such as cold, infection and others, creates the micro-focus of hypothermia in the prostate tissue. In order to eliminate this focus of micro-hypothermia, blood flow increases through spontaneous expansion of the capillary network locally. The nervous system does not involve in it, so the expansion can continue and continue. Formation of new capillaries is responsible for the growth of the prostate tissue [16].

\section{DATD, as Safe and Effective Treatment for BPH}

DATD facilitates the treatment of the affected prostate by topical application of a special blend of waxes, which can collect the natural radiation of body heat, thus becoming a source of energy. This special wax mixture is called a thermoelement. DATD also consist of an elastic belt that holds the thermoelement in projection of the prostate gland for a long time. Thus, the thermoelement acts as a natural source of heat for the prostate. The neoprene belt keeps the thermoelement tight to the tailbone area and prevents heat disappearance (Figure 2). DATD by spreading energy towards local micro-hypothermia in the prostate tissue terminates it, stopping enlargement gradually. This leads to the elimination of pain and urine symptoms. $[17,18]$.

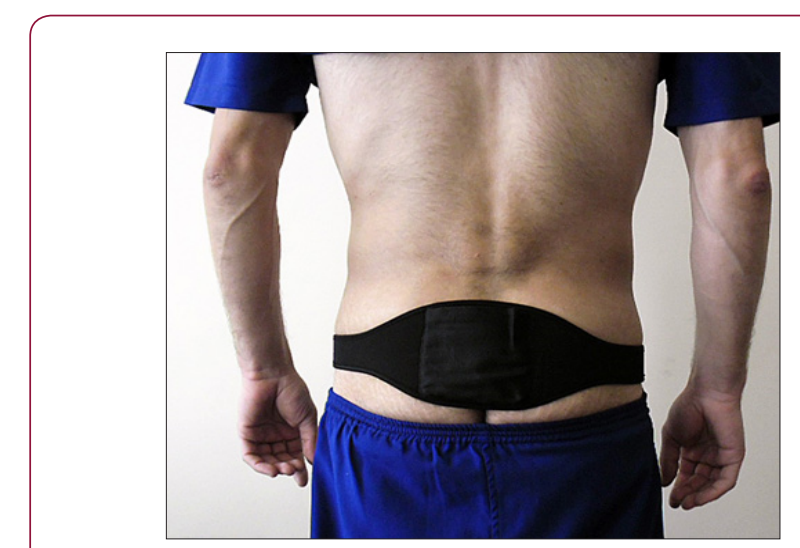

Figure 2: DATD tightly attaches thermoelement to the coccyx area for a prolonged period of time.

\section{Cost-Effectiveness of DATD}

In the past decade, significant progress has been also made in understanding the demographics of BPH. The prevalence of this condition is increasing with the population aging and so does the economic burden, which is comprised of 3 components: direct costs for its treatment, indirect - lost earning and intangible costs - pain and suffering. In a rough estimate, the cost of treating BPH in the United States is $\$ 4$ billion per year [19]. The investigation in Europe of annual cost of BPH medical treatment was lowest in the UK about $\$ 1000$ and highest in Poland approximately \$1500 [20]. DATD is one-time purchase for $\$ 200$ that makes TT the most cost-effective therapy and in addition TT significantly improves QoL of men with BPH without side effects.

\section{Summary and Conclusion}

"Watchful waiting", which was introduced about 20 years ago in the guidelines for doctors to protect men with BPH from side events, which follow up the use of conventional BPH drugs and 
operations. DATD has shown to be effective, safe and cost-effective for men with BPH [21]. TT with DATD is a completely new treatment in the world, therefore, the use of TT with DATD should rely on a strong collaboration between a patient, primary care physician and urologist [22]. Thus, TT and DATD should be recommended as monotherapy for LUTS / BPH, affecting millions of men around the world. In the guidelines for doctors, "watchful waiting" for men with BPH should be replaced with Thermobalancing therapy.

\section{Acknowledgment}

I am grateful to Prof Aghajanyan IG, the founder of Armenian Association of Urologists, for conducting the clinical studies on Thermobalancing therapy.

\section{References}

1. Unnikrishnan R, Almassi N, Fareed K (2017) Benign prostatic hyperplasia: Evaluation and medical management in primary care. Cleve Clin J Med 84(1): 53-64.

2. Blankstein U, Van Asseldonk B, Elterman DS (2016) BPH update: medical versus interventional management. Can J Urol 23(1): 10-15.

3. Lim KB (2017) Epidemiology of clinical benign prostatic hyperplasia. Asian Journal of Urology 4(3): 148-151.

4. Allen S, Aghajanyan IG (2015) Benign Prostatic Hyperplasia Treatment with New Physiotherapeutic Device. Urol J 12(5): 2371-2376.

5. Allen S, Adjani A (2016) Therapeutic Device and Method, United States Patent and Trademark Office. Patent, US 9(408): 744 B2.

6. Gratzke C, Bachmann A, Descazeaud A, Drake MJ, Madersbacher S, et al. (2015) EAU guidelines on the assessment of non-neurogenic male lower urinary tract symptoms including benign prostatic obstruction. Eur Urol 67(6): 1099-1109.

7. Voznesensky IE, Shaw KJD, Yafi F, Hellstrom WJG (2017) Benign prostatic hyperplasia treatment options and their effects on sexual function. Sex Med Rev 5(1): 87-102.

8. Elshal AM, Elmansy HM, Elkoushy MA, Elhilali MM (2012) Male sexual function outcome after three laser prostate surgical techniques: a single center perspective. Urology 80(5): 1098-1104.

9. Ronis MJJ, Pedersen KB, Watt J (2018) Adverse effects of nutraceuticals and dietary supplements. Annu Rev Pharmacol Toxicol 58: 583-601.

10. Corona, G, Vignozzi L, Rastrelli G, Francesco Lotti, Sarah Cipriani, et al. (2014) Benign prostatic hyperplasia: a new metabolic disease of the aging male and its correlation with sexual dysfunctions. Int J Endocrinol ID 329456.

11. Allen S (2016) The Origin of Chronic Diseases Can Be in Capillary Pathology: An Evidence from Clinical Trials on Thermobalancing Treatment of Prostate Reveals. Achiev Life Sci 10: 197-202.

12. Aghajanyan IG, Allen S (2016) Positive Response to Thermobalancing Therapy Enabled by Therapeutic Device in Men with Non-Malignant Prostate Diseases: BPH and Chronic Prostatitis. Diseases 4(2): 18.

13. Shimizu S1, Tsounapi P, Shimizu T, Honda M, Inoue K, et al. (2014) Lower urinary tract symptoms, benign prostatic hyperplasia/benign prostatic enlargement and erectile dysfunction: Are these conditions related to vascular dysfunction? Int J Urol 21(9): 856-864.

14. Saito M, Tsounapi P, Oikawa R, Shimizu S, Honda M, et al. (2014) Prostatic ischemia induces ventral prostatic hyperplasia in the SHR; possible mechanism of development of BPH. Sci Rep 4: 3822.

15. Thurmond P, Yang JH, Azadzoi KM (2016) LUTS in pelvic ischemia: a new concept in voiding dysfunction. Am J Physiol Renal Physiol 310(8): F738-F743.

16. Allen S, Aghajanyan IG (2016) Use of thermobalancing therapy in ageing male with benign prostatic hyperplasia with a focus on etiology and pathophysiology, Aging Male 20(1): 1-5.

17. Allen S (2017) The Vascular Factor Plays the Main Role in the Cause of Pain in Men with Chronic Prostatitis and Chronic Pelvic Pain Syndrome: The Results of Clinical Trial on Thermobalancing Therapy. Diseases 5(4): 25.

18. Allen S, Aghajanyan IG (2016) Thermobalancing conservative treatment for moderate-to-low-degree lower urinary tract symptoms (LUTS) secondary to prostate enlargement. Cogent Medicine 3(1): 1195067.

19. Vuichoud C, Loughlin KR (2015) Benign prostatic hyperplasia: epidemiology, economics and evaluation. Can J Urol 22(1): 1-6.

20.Speakman M, Kirby R, Doyle S, Ioannou C (2015) Burden of male lower urinary tract symptoms (LUTS) suggestive of benign prostatic hyperplasia (BPH) - Focus on the UK. BJU Int 115(4): 508-519.

21. Allen S (2018) Innovative Thermobalancing therapy can help millions of men with enlarged prostate gland to improve the quality of life and wellbeing throughout the world. World Scientific News (WSN) 105: 51-61.

22. Allen S (2018) Dr Allen's Therapeutic Devices Should be Implemented in the Healthcare System for the Treatment of Chronic Noncancerous Prostate and Kidney Diseases Saving People's Well-Being and Money. Ann Mil Health Sci Res 16(2): e81033.

\section{ISSN: 2574-1241}

DOI: $10.26717 / B J S T R .2018 .10 .001968$

Allen Simon. Biomed J Sci \& Tech Res

(C) (i) This work is licensed under Creative Commons Attribution 4.0 License

Submission Link: https://biomedres.us/submit-manuscript.php

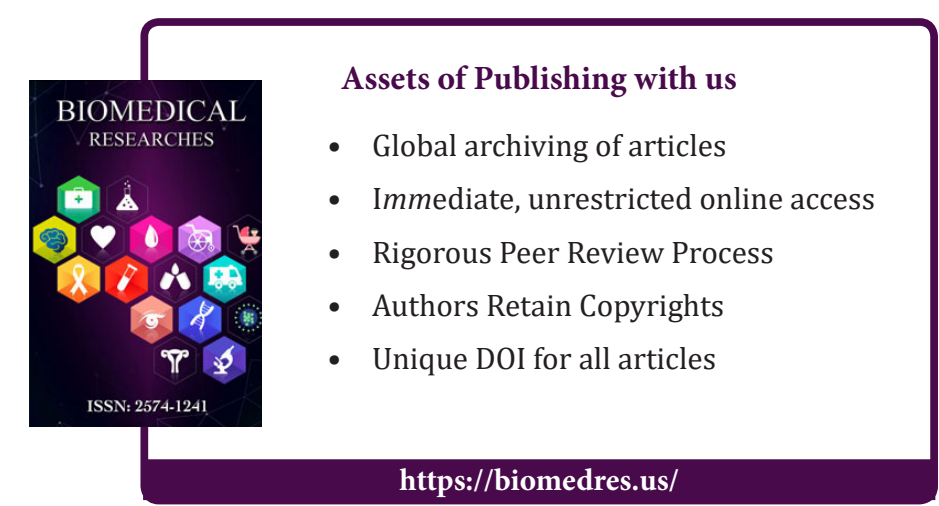

